\title{
A parallel algorithm of non-linear high-order boundary condition problem using form initial condition problem
}

\author{
Chen Zhao, Zhang Shesheng, Li Yuguang* ${ }^{1}$ \\ Association of Mathematical Modeling, Wuhan University of Technology, Wuhan, 430070,China \\ ${ }^{a}$ Corresponding author: Li Yuguang, Liyuguang@qq.com
}

Keywords: boundary condition problem; initial condition problem;parallel algorithm.

\begin{abstract}
The non-linear high-order boundary condition problem is transformed into form initial condition problem with few parameters, and the steps of calculating the non-linear parameter is constructed. The boundary condition problem is solved using parallel computer. Comparing with the result of boundary condition problem, the examples show that the numerical time is observably decreasing by proposed algorithm.
\end{abstract}

\section{Introduction}

We often meet non-linear high-order boundary condition problems, such as the hull deformation problem. But few of them can get the analytic solution; most of them are solved by numerical methods. Because problem is non-linear and high order, it is often spending long CPU time for numerical method. Zha[1]gave a survey and classification of various methods in numerical solution of the boundary value problems, and pointed that methods of initial condition also spent more CPU time. Dua[2]concerned with third-order non-linear ordinary differential equation, and gave existence result by using a priori estimate. Regan[3]also discussed third-order boundary value problems, and gave some way about application. By using non-polynomial spline technique, Abd[4]calculated linear third-order boundary value problems. He showed that long CPU time was needed, if the problem is non-linear. Zhang[5]converted two-order boundary differential equation into a form of initial problem, and got the solution by using Runge-Kutta numerical method. Its convergence theory was given by Wei[6].

In this paper, the nth-order non-linear boundary value problem is converted to initial value problem with zero initial condition. The examples of two-order and four-order problem are given. .

\section{2 n-order non-linear boundary value problem}

Suppose that the n-order non-linear boundary value problem as

$$
\begin{aligned}
& y^{(n)}=f\left(x, y^{\prime}, y^{\prime \prime}, \ldots, y^{(n-1)}\right) \\
& y^{(n i)}(a)=y^{(n i)}{ }_{a} \quad i=1,2, \ldots, k_{a}
\end{aligned}
$$

By using the transform $\mathrm{z}=(\mathrm{x}-\mathrm{a}) /(\mathrm{b}-\mathrm{a})$, then $\mathrm{z}$ is taken value in $[0,1]$. So we could just consider the value of $\mathrm{x}$ in $[0,1]$.

While $a=0, b=1$, above problem can be written as

$$
\begin{aligned}
& y^{(n)}=f\left(x, y^{\prime}, y^{\prime \prime}, \ldots, y^{(n-1)}\right) \quad y^{(n-n i)}(0)=y_{a}^{(n-n i)} \quad i=1,2, \ldots, k_{a} \\
& y^{(n-n j)}(1)=y_{b}^{(n-n j)} \quad j=1,2, \ldots, k_{b}
\end{aligned}
$$

Because the $y=y(x)$ is the function of $x$, then $y(k), k=0,1, . ., n$ is also the function of $x$, so we credited as

$$
y^{(n)}=F_{0}(x) \quad F_{0}(x)=f\left(x, y^{\prime}, y^{\prime \prime}, \ldots, y^{(n-1)}\right)
$$

\footnotetext{
${ }^{1}$ Corresponding author: Li Yuguang, Liyuguang@qq.com.
} 
It is easy to show the following theorem:

Theorem 1:

Suppose that $\mathrm{F} 0(\mathrm{x})$ is Continuous in $[0,1]$, then

$$
y^{(n-k)}=c_{n-k}+\ldots \frac{c_{n-1}}{(k-1) !} x^{k-1}+F_{k}(x) \quad k=1, \ldots, n
$$

Where

$$
F_{k}(x)=\int_{0}^{x} F_{k-1}(x) d x
$$

By applying the boundary conditions,

$$
y_{a}^{(n i)}=c_{n i} \quad i=1,2, \ldots, k_{a} \quad y_{b}^{(n-n j)}=c_{n-n j}+\ldots+\frac{c_{n-1}}{\left(n_{j}-1\right) !}+F_{k}(1) \quad j=1,2, \ldots, k_{b}
$$

the constant $\mathrm{C}=(\mathrm{c} 0, \mathrm{c} 1, \ldots, \mathrm{cn}-1)$ can be found from above equations.

Theorem 2:

Supposed that $\mathrm{F} 0(\mathrm{x})$ is Continuous on $[0,1]$, then the solution of the equation (2.1) is equivalent to the solution of the following initial value problem

$$
F_{k}^{\prime}(x)=F_{k-1}(x) \quad k=1,2, . ., n \quad F_{k}(0)=0
$$

Proof:

Since that $\mathrm{F} 0(\mathrm{x})$ is Continuous on $[0,1]$, then $\mathrm{F} 1^{\prime}=\mathrm{F} 0, \mathrm{~F} 2^{\prime}=\mathrm{F} 1$, so the Second derivative of $\mathrm{F} 2$ exists and can be written as F2', and F2'"=F'1=F0 . Similarly, we can proof that the n-order derivative of $\mathrm{Fn}$ is exists, and $\mathrm{Fn}(\mathrm{n})=\mathrm{F} 0=\mathrm{y}(\mathrm{n})$. This shows the solution of (2.1) is the one of $\mathrm{Eq}(2.7)$ with $\operatorname{Eq}(2.4)$.

\section{The Second derivative}

While the order of derivative $n=2$, then

$$
y^{(2)}=f\left(x, y, y^{\prime}\right) \quad y(a)=y_{a} \quad y(b)=y_{b}
$$

Making the function of $\mathrm{y}$ is

$$
y=c_{0}+c_{1}(x-a)+F_{2}(x)
$$

Where

$$
F_{2}^{\prime}(x)=F_{1}(x) \quad F_{1}^{\prime}(x)=F_{0}(x) \quad F_{1}(a)=0 \quad F_{2}(a)=0
$$

By using boundary conditions, we have

$$
c_{0}=y_{a} \quad c_{1}=\left[y_{b}-F_{2}(b)\right] /(b-a)
$$

Example 1

Consider non-linear boundary value problem:

$$
y^{\prime \prime}=\frac{1}{1+x+y^{2}+y^{\prime 2}}+2-\frac{1}{1+x+x^{4}+4 x^{2}} \quad y(0)=0 \quad y(1)=0
$$


Divide domain $[0,1]$ to $N$ subdomain, the step length $h=1 / N$, the error limit $\varepsilon=0.0001$, and initial value $\mathrm{c} 1=0$, the results calculated are shown in Table 1 , which $\mathrm{k} 1$ is the number of iterations of our method. When $\mathrm{N}>80$, only 2 iterations to meet the convergence conditions. The k2 in Table 1 is the number of iterations using the Gauss-Seidel method to solve equations for the same step length. The equations are given by difference method of the boundary value problem. From the Table 1, when $\mathrm{N}$ increases, the number of iterations of the Gauss-Seidel method increases rapidly.

TABLE I. The relationship between the number of iterations and the number of nodes $\mathbf{N}$

\begin{tabular}{llllllll}
\hline $\mathrm{N}$ & 5 & 10 & 15 & 20 & 40 & 80 & 160 \\
\hline $\mathrm{k}_{1}$ & 4 & 4 & 4 & 3 & 3 & 2 & 2 \\
$\mathrm{~K}$ & 14 & 65 & 112 & 198 & & & \\
2 & & & & & & & \\
\hline
\end{tabular}

Because the controlling equations are non-linearity and the calculations of solving equations by Gauss-Seidel method are arduous, we use domain decomposition parallel computing method to solve the boundary value problems. Parallel computing, we first divide the region [0,1] into k sub-regions of equal length, and each sub-region is arranged for a processor to compute. Each iteration, exchange the boundary function's value of the adjacent region, and then continue the iteration until meeting the convergence conditions. In this article, we take 16 processors to compute.

\section{The fourth derivative}

While the order of derivative $n=4$, then

$$
y^{(4)}=f\left(x, y, y^{\prime}, y^{\prime \prime}, y^{\prime \prime \prime}\right) \quad y(0)=y_{a} \quad y(b)=y_{b} \quad y^{\prime \prime}(0)=y^{\prime \prime}{ }_{a} \quad y^{\prime \prime}(b)=y^{\prime \prime}{ }_{b}
$$

Making the function is

$$
\begin{aligned}
& F_{0}(x)=f\left(x, y(x), y^{\prime}(x), y^{\prime \prime}(x), y^{\prime \prime}(x), y^{\prime \prime}(x)\right) \\
& F_{k}{ }^{\prime}(x)=F_{k-1}(x) \quad F_{k}(0)=0 \quad k=1,2,3,4
\end{aligned}
$$

Then

$$
\begin{aligned}
& y^{\prime \prime}=c_{3}+F_{1}(x) \quad y^{\prime \prime}=c_{2}+c_{3} x+F_{2}(x) \\
& y^{\prime}=c_{1}+c_{2} x+\frac{c_{3}}{2} x^{2}+F_{3}(x) \quad y=c_{0}+c_{1} x+\frac{c_{2}}{2} x^{2}+\frac{c_{3}}{6} x^{3}+F_{4}(x)
\end{aligned}
$$

By using the boundary conditions, we have

$$
\begin{aligned}
& c_{0}=y_{a} \quad c_{2}=y^{\prime \prime}{ }_{a} \quad c_{3}=\left[y^{\prime \prime}{ }_{b}-c_{2}-F_{2}(b)\right] / b \\
& c_{1}=\left[y_{b}-c_{0}-\frac{c_{2}}{2} b^{2}-\frac{c_{3}}{6} b^{3}-F_{4}(b)\right] / b
\end{aligned}
$$

Because the value of the boundary conditions are zero, so

$$
\begin{aligned}
& c_{0}=0 \quad c_{2}=0 \quad c_{3}=-F_{2}(b) / b \\
& c_{1}=\left[-\frac{c_{3}}{6} b^{3}-F_{4}(b)\right] / b=-\frac{c_{3}}{6} b^{2}-F_{4}(b) / b
\end{aligned}
$$

Then the value of the function is

$$
y=c_{1} x+\frac{c_{3}}{6} x^{3}+F_{4}(x)
$$

Which F4 is calculated by the following initial value problems 


$$
\begin{aligned}
& F_{k}{ }^{\prime}(x)=F_{k-1}(x) \quad F_{k}(0)=0 \quad k=1,2,3,4 \\
& F_{0}(x)=f\left(x, y(x), y^{\prime}(x), y^{\prime \prime}(x), y^{\prime \prime}(x), y^{\prime \prime \prime}(x)\right)
\end{aligned}
$$

Example 2

Consider the non-linear boundary value problem with fourth derivative:

$$
\begin{aligned}
& y^{(4)}=\frac{a}{1+y^{2}+y^{\prime \prime 2}}-\frac{a}{1+2 \sin ^{2}(x)}+\sin (x) \quad 0<x<\pi \\
& y(0)=0 \quad y(\pi)=0 \quad y^{\prime \prime}(0)=0 \quad y^{\prime \prime}(\pi)=0
\end{aligned}
$$

According to the above method, we could get

$$
y=c_{1} x+\frac{c_{3}}{6} x^{3}+F_{4}(x) \quad y^{\prime \prime}=c_{3} x+F_{2}(x)
$$

Take the parameters $\mathrm{a}=0.1$, the number of nodes $\mathrm{N}=40$, and the initial value $\mathrm{c} 1=\mathrm{c} 3=0$, the relationship between the value of constant c1 and c3 and the number of iterations $\mathrm{M}$ is shown in Table 2. Using the Four-order Runge-Kutta method, firstly, obtains Fk and y, k=1,2,3,4 by the initial value problem; then calculates the new value of c1 and c3, continue the iteration like this to calculate the value of c1 and c3. From the Table 2, when the number of iteration $M>1$, the two iterative values of constant c1 and c3 are less than 0.01 . The analytical solution of this example is $y=\sin (x)$.

TABLE II. The relationship between the value of constant c1, c3 and the number of iterations $\mathrm{M}$

\begin{tabular}{llllll}
\hline $\mathrm{M}$ & 0 & 1 & 2 & 3 & 4 \\
\hline $\mathrm{c}_{1}$ & 0 & 1.0401 & 1.007 & 1.0098 & 1.0096 \\
$\mathrm{C}_{3}$ & 0 & -1.0309 & -0.98896 & -0.99165 & -0.99144 \\
\hline
\end{tabular}

\section{Summary}

In this paper, we transform the non-linear high-order boundary condition problem into initial condition problem. The calculation of non-linear function is converted to the calculation of non-linear parameter. By using initial value problem, the iteration steps of calculating the non-linear parameter are constructed. The second-order boundary condition problem is calculated by parallel algorithm, and comparing with the result of initial condition problem is given. We find that the convergence speed of the initial condition problem is much higher than the boundary condition problem..

\section{ACKNOWLEDGMENT}

The paper is financially supported by China national natural science foundation (No.51139005).

\section{References}

[1] Xin Chen, XinCong Zhou, Shesheng Zhang, Dan Li, A numerical fluid-solid coupling model for the dynamics of ships in atrocious sea conditions $[\mathrm{J}]$, Journal of Algorithms \& Computational Technology,2015, Vol. 9 No. 2, 163-175.

[2] Zengji Dua, Weigao Gea, Xiaojie Linb, Existence of solutions for a class of third-order nonlinear boundary value problems[J] .Journal of Mathematical Analysis and Applications, Vol.294, No.1, 2004, $104-112$.

[3] Regan D . Topological transversality : Applications of third order boundary value problems[J], Siam J Math Anal, 1987, 18, 630-641.

[4] F.A. Abd El-Salam, A.A. El-Sabbagh and Z.A. ZAki, The numerical solution of linear third order boundary value problems using non-polynomial spline technique $[\mathrm{J}]$, Journal of American Science, 2010(6), 303-309. 
[5] Shesheng Zhang, Jianing Wei, A mathematical model solving boundary condition problem using algorithm of the initial value problem[J], J. Wuhan Transportation University, 1999(5), 504-507.

[6] Jianing Wei, Shesheng Zhang, Yakup Paker, A high order numerical model of non-linear boundary equation[J], J Naval University of Engineering, 2001(3), 7-10.. 